\title{
Massive bulges are not just ellipticals surrounded by disks
}

\author{
Dimitri A. Gadotti \\ European Southern Observatory \\ email: dgadotti@eso.org
}

\begin{abstract}
Using results from parametric multi-component multi-band image fitting of 1000 local massive galaxies in the SDSS, I investigate scaling relations of elliptical galaxies and bulges of disk galaxies. I show that ellipticals and bulges occupy different loci in both the edge-on and face-on views of the fundamental plane. In addition, ellipticals and bulges have offset mass-size relations (see Fig. 1). These results imply that massive bulges are not just massive ellipticals with a surrounding disk, a misconception driven by early studies. This is evidence that massive ellipticals and bulges have different formation histories, with important consequences for studies on galaxy formation and evolution. Full details can be seen in Gadotti (2009).
\end{abstract}

Keywords. galaxies: bulges, galaxies: elliptical and lenticular, $\mathrm{cD}$, galaxies: evolution, galaxies: formation, galaxies: structure

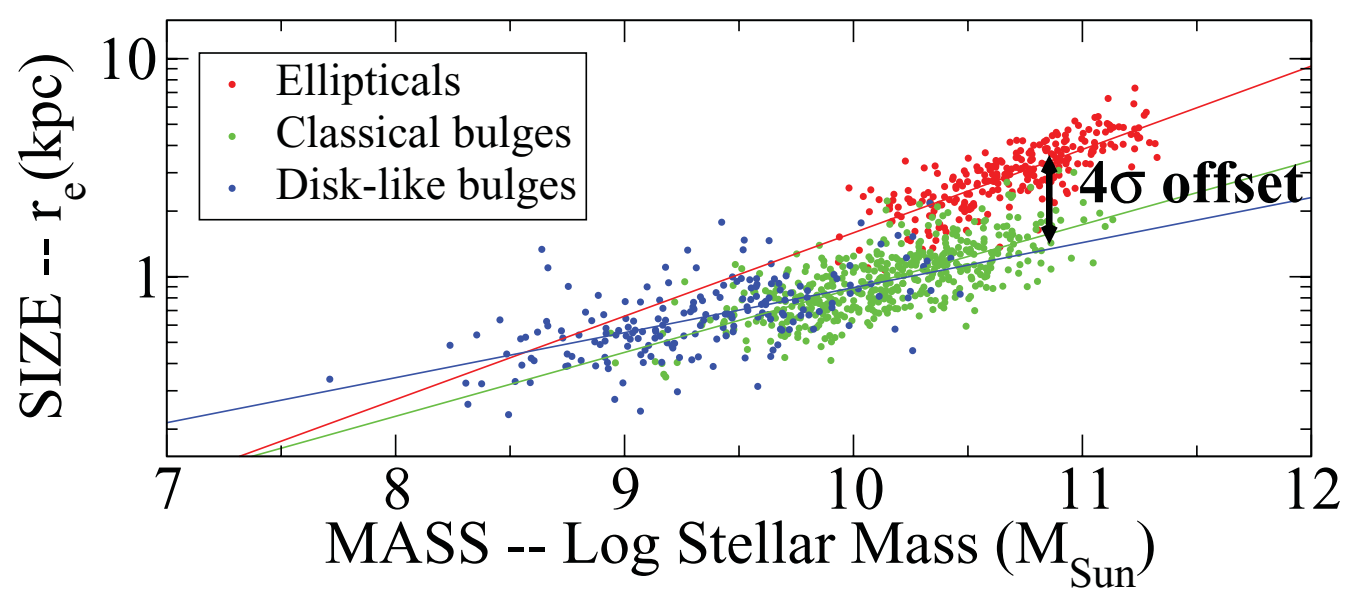

Figure 1. Mass-size relations of elliptical galaxies, classical bulges and disk-like bulges, as indicated. The relations for massive ellipticals and classical bulges in disk galaxies show a clear offset at a $4 \sigma$ confidence level. If one puts a disk surrounding a massive elliptical galaxy (i.e. with $\mathrm{M}_{\star} \geqslant 10^{10} \mathrm{M}_{\odot}$ ) one does not end up with a disk galaxy with a bulge similar to those observed in Nature (adapted from Gadotti 2009).

\section{References}

Gadotti, D. A. 2009, MNRAS, 393, 1531 